\title{
Serum Lactate Dehydrogenase Level as a Prognostic Factor for COVID-19: A Retrospective Study Based on a Large Sample Size
}

\section{OPEN ACCESS}

Edited by:

Constantinos Tsioutis,

European University Cyprus, Cyprus

Reviewed by:

Diansan Su,

Shanghai JiaoTong University, China

Qiaobing Huang,

Southern Medical University, China

Zhenhua Zeng,

Southern Medical University, China

${ }^{*}$ Correspondence:

Xiaohui Wu

wuxiaohui1971@sina.com

Weiwei Yuan

53906199@qq.com

Kuan Luo

luokuan@163.com

†These authors have contributed equally to this work

Specialty section

This article was submitted to Infectious Diseases - Surveillance,

Prevention and Treatment

a section of the journal

Frontiers in Medicine

Received: 24 February 2021 Accepted: 29 November 2021

Published: 04 January 2022

Citation:

Huang Y, Guo L, Chen J, Wu M,

Zhang C, Liu Z, Li J, Li K, Xiong Z,

Wu Q, Li Z, Luo K, Yuan W and Wu X

(2022) Serum Lactate Dehydrogenase

Level as a Prognostic Factor for

COVID-19: A Retrospective Study

Based on a Large Sample Size.

Front. Med. 8:671667.

doi: 10.3389/fmed.2021.671667

\begin{abstract}
Yihui Huang ${ }^{1+}$, Liang Guo ${ }^{1+}$, Jiwei Chen ${ }^{2+}$, Meng Wu ${ }^{3+}$, Chao Zhang ${ }^{4+}$, Zeming Liu ${ }^{5}$, Jinpeng $\mathrm{Li}^{2}$, Kun $\mathrm{Li}^{6}$, Zhongwei Xiong ${ }^{7}$, Qian $\mathrm{Wu}^{7}$, Zhengwei $\mathrm{Li}^{7}$, Kuan $\mathrm{Luo}^{8 *}$, Weiwei Yuan $^{9 *}$ and Xiaohui $\mathrm{Wu}^{7 *}$
\end{abstract}

\begin{abstract}
${ }^{1}$ Department of Plastic Surgery, Zhongnan Hospital of Wuhan University, Wuhan, China, ${ }^{2}$ Department of Thyroid and Breast Surgery, Zhongnan Hospital of Wuhan University, Wuhan, China, ${ }^{3}$ Department of Ultrasound, Zhongnan Hospital of Wuhan University, Wuhan, China, ${ }^{4}$ Department of Cardiovascular Surgery, Tongji Medical College, Union Hospital, Huazhong University of Science and Technology, Wuhan, China, ${ }^{5}$ Department of Plastic Surgery, Tongji Hospital, Tongji Medical College, Huazhong University of Science and Technology, Wuhan, China, ${ }^{6}$ Department of Hepatobiliary and Pancreatic Surgery, Zhongnan Hospital of Wuhan University, Wuhan, China, ${ }^{7}$ Department of Neurosurgery, Zhongnan Hospital of Wuhan University, Wuhan, China, ${ }^{8}$ Department of Neurosurgery, Wuhan Puren Hospital, Wuhan, China, ${ }^{9}$ Department of Plastic and Cosmetic Surgery, Shenzhen People's Hospital (The Second Clinical Medical College, Jinan University, The First Affiliated Hospital, Southern University of Science and Technology), Shenzhen, China
\end{abstract}

Background: In this study, we investigated the relationship between serum lactate dehydrogenase ( $\mathrm{LDH}$ ) level and disease progression and prognosis of patients with COVID-19.

Methods: We retrospectively reviewed the information of 1,751 patients with COVID-19 from Leishenshan Hospital in Wuhan, China. Univariate and multivariate Cox regression analyses as well as Logistics regression analyses, and Kaplan-Meier curves were used to determine the association between LDH levels and the prognosis of COVID-19 patients.

Results: LDH was an independent risk factor for in-hospital death no matter it was taken as classified variable and continuous variable (all $P=0.001$ ) but not for severe or critical illness status. The Kaplan-Meier curves for LDH level showed that an elevated level of LDH was associated with in-hospital death.

Conclusions: In patients with COVID-19, the increased LDH level is associated with a higher risk of negative clinical prognosis and higher mortality. This will provide a reference for clinicians and researchers to understand, diagnose, and treat patients with COVID-19. Further prospective studies with larger sample sizes are needed to verify these findings.

Keywords: COVID-19, lactate dehydrogenase (LDH), SARS-CoV-2, prognostic factor, Leishenshan Hospital

\section{INTRODUCTION}

The world is currently experiencing a major coronavirus disease 2019 (COVID-19) pandemic (1-3). Although COVID-19 can cause severe illness, the case fatality rate is relatively low (4). As of 22 April 2020, more than 2,500,000 cases were reported worldwide, with more than 170,000 deaths. Leshenshan Hospital is hosted by Zhongnan Hospital and is a temporary, specialized 1,600-bed hospital designated for the treatment of patients with COVID-19. From February 8, 2020 to 
April 15, 2020, 1,880 patients with confirmed COVID-19 were admitted. Lactate dehydrogenase $(\mathrm{LDH})$ is one of the enzymes of the glycolytic pathway that catalyzes the conversion of pyruvate to lactate with concurrent conversion of reduced nicotinamide adenine dinucleotide (NADH) to nicotinamide adenine dinucleotide (NAD) (5). Elevated $\mathrm{LDH}$ levels have been shown to be associated with more severe disease and increased mortality in multiple diseases (5-7).

Clinicians and researchers have been making efforts to understand and cure COVID-19; however, knowledge of its pathogenesis is limited (8-10). In our study, we investigated the effect of serum $\mathrm{LDH}$ levels on the disease progression and prognosis of patients with COVID-19. There is usually a normal range for the measurement of $\mathrm{LDH}$ in clinical application. The study group assignment in this study was generated based on the normal range of $\mathrm{LDH}$ level but we also took $\mathrm{LDH}$ as a continuous variable when conducting analyses so that we can intuitionally detect the relation between $\mathrm{LDH}$ level and the prognosis of COVID-19 patients.

\section{MATERIALS AND METHODS}

\section{Study Design and Patients}

We conducted a retrospective cohort study of 1,880 patients with laboratory-confirmed SARS-CoV-2 infection, who were admitted to Leishenshan Hospital in Wuhan, China, with COVID-19 between February 9 and March 18, 2020. The medical records of these patients were reviewed by two experienced physicians, and detailed information on patient demographics, clinical features, laboratory test results, computed tomography (CT) images, and treatment were extracted. A total of 129 patients who did not have an $\mathrm{LDH}$ test or whose $\mathrm{LDH}$ test results were missing were excluded, leaving 1,751 patients for the analysis. Of these patients, 1,653 had an LDH level within the normal range (125-343 U/L), 43 had a low LDH $(<125$ $\mathrm{U} / \mathrm{L}$ ), and 55 had an elevated $\mathrm{LDH}(>343 \mathrm{U} / \mathrm{L})$. Considering the clinical implications of the $\mathrm{LDH}$ results, patients with a normal or decreased $\mathrm{LDH}$ level were assigned to one group and compared with the 55 patients with an elevated $\mathrm{LDH}$ level. $\mathrm{LDH}$ was also taken as a continuous variable when conducting the analyses for the prognosis of COVID-19 patients. All the laboratory findings were baseline data including LDH level.

\section{Definitions}

The primary outcome in this study was the occurrence of death during the period of hospitalization. The illness status was defined according to the seventh edition of the Chinese management guideline for COVID-19 published by the Chinese National Health Commission (11). We acquired records of the illness status on admission and the highest level of illness status of patients during their hospitalization. The latter was also used as an outcome in this study. Mild and common cases were assigned in one group while severe and critical cases were combined into one group when illness status was used as an outcome in analysis. The survival time in this study was defined as the period from the day that patients on admission to the day deaths occurred or follow-up stopped and it was described as "follow-up days." An axillary temperature over $37.3^{\circ} \mathrm{C}$ was defined as fever. A semi-quantitative score system based on the results of the CT images was generated to evaluate the pulmonary lesions of COVID-19 patients. Each of the image characteristics including ground-glass opacities, reticulation or cords change, consolidation, and pleural effusions were assigned 1 point. Score 1 was the sum of the points mentioned above. Score 2 was assigned based on the area of the lung lobes involved: no involvement (0 points); $<25 \%$ involvement (1 point); $26-50 \%$ ( 2 points); $51-75 \%$ (3 points); $>75 \%$ ( 4 points). The total score was equal to the sum of score 1 and score 2.

\section{Ethics Approval and Patient Consent}

This study obtained the approval of the Research Ethics Commission of the Zhongnan Hospital of Wuhan University (approval number: 2020074). The requirement for informed consent was waived because the study was retrospective.

\section{Statistical Analysis}

All the statistical analyses were performed using SPSS Version 23.0 (IBM Corp, Armonk, NY, USA). Comparisons between the low/normal and elevated LDH level groups for categorical data were performed using the chi-square test or Fisher's exact test if the number of observations was limited. Comparisons of continuous variables were performed using independent group $t$-tests when the data were normally distributed, or Mann-Whitney $U$ test when the data were not normally distributed. Univariate and multivariate Cox regression analyses were conducted for investigating the relation between inhospital death and LDH level, while Logistics regression analyses were generated for detecting the relation between illness status and LDH level. Factors which were significant associated with primary outcomes in univariate analyses were selected into adjustment when conducting multivariate analyses. For intuitionally detecting the relation between $\mathrm{LDH}$ level and the prognosis of COVID-19 patients, LDH level was taken as both classified variable and continuous variable in the regression analyses. Based on the result of regression analyses, KaplanMeier survival analyses were used to explore whether LDH levels were associated with prognosis. Curve fitting analysis was performed to assess the relation between CT performances and survival time. Two-sided $p$-values $<0.05$ were regarded as statistically significant.

\section{RESULTS}

\section{Demographics, Clinical Information, and Treatment}

The mean age of the patients in the elevated $\mathrm{LDH}$ group was $63.66 \pm 14.49$ years, which was higher than that in the normal/decreased LDH group (57.51 $\pm 14.36, P=0.002$; Table 1). Severe and critical cases account for a major part of patients with elevated $\mathrm{LDH}$ level no matter on 
TABLE 1 | Demographic and clinical information for COVID-19 patients in different LDH level.

\begin{tabular}{|c|c|c|c|c|c|}
\hline \multirow{2}{*}{$\begin{array}{l}\text { Covariate } \\
\text { Age, year, mean } \pm S D\end{array}$} & \multicolumn{2}{|c|}{ LDH normal or decreased group $(n=1,696)$} & \multicolumn{2}{|c|}{ LDH elevated group $(n=55)$} & \multirow{2}{*}{$\begin{array}{c}\boldsymbol{P} \text {-value } \\
0.002\end{array}$} \\
\hline & \multicolumn{2}{|c|}{$57.51 \pm 14.36$} & \multicolumn{2}{|c|}{$63.66 \pm 14.49$} & \\
\hline \multicolumn{6}{|l|}{ Sex } \\
\hline Female & 890 & $52.50 \%$ & 26 & $47.30 \%$ & 0.447 \\
\hline Male & 806 & $47.50 \%$ & 29 & $52.70 \%$ & \\
\hline Comorbidity & 487 & $60.30 \%$ & 36 & $73.50 \%$ & 0.066 \\
\hline Cardiovascular disease & 331 & $41.00 \%$ & 22 & $44.90 \%$ & 0.587 \\
\hline Pulmonary disease & 82 & $10.60 \%$ & 5 & $13.90 \%$ & 0.533 \\
\hline Nervous system disease & 50 & $6.20 \%$ & 4 & $8.20 \%$ & 0.581 \\
\hline Endocrine disease & 129 & $16.00 \%$ & 6 & $12.20 \%$ & 0.488 \\
\hline Malignancy & 56 & $6.90 \%$ & 3 & $6.10 \%$ & 0.828 \\
\hline Digestive system disease & 41 & $5.10 \%$ & 4 & $8.20 \%$ & 0.347 \\
\hline \multicolumn{6}{|c|}{ Illness status of COVID-19 on admission } \\
\hline Mild & 649 & $38.30 \%$ & 14 & $25.50 \%$ & $<0.001$ \\
\hline Common & 770 & $45.40 \%$ & 14 & $25.50 \%$ & \\
\hline Severe & 260 & $15.30 \%$ & 20 & $36.40 \%$ & \\
\hline Critical & 17 & $1.00 \%$ & 7 & $12.70 \%$ & \\
\hline \multicolumn{6}{|c|}{ The highest level of illness status at hospitalization } \\
\hline Mild and common & 903 & $53.40 \%$ & 5 & $9.30 \%$ & $<0.001$ \\
\hline Severe & 756 & $44.70 \%$ & 36 & $66.70 \%$ & \\
\hline Critical & 33 & $2.00 \%$ & 13 & $24.10 \%$ & \\
\hline \multicolumn{6}{|c|}{ The highest level of oxygen support } \\
\hline Low flow oxygen therapy & 250 & $86.20 \%$ & 6 & $33.30 \%$ & $<0.001$ \\
\hline High flow oxygen therapy & 39 & $13.40 \%$ & 7 & $38.90 \%$ & \\
\hline Tracheal intubation & 1 & $0.30 \%$ & 4 & $22.20 \%$ & \\
\hline ECMO & 0 & $0.00 \%$ & 1 & $5.60 \%$ & \\
\hline \multicolumn{6}{|c|}{ Symptoms when admitted to the hospital } \\
\hline Fever or myalgia & 575 & $79.00 \%$ & 35 & $79.50 \%$ & 0.929 \\
\hline Respiratory system symptoms & 588 & $80.80 \%$ & 35 & $79.50 \%$ & 0.842 \\
\hline Digestive system symptoms & 74 & $10.20 \%$ & 6 & $13.60 \%$ & 0.444 \\
\hline Nervous system symptoms & 24 & $3.30 \%$ & 2 & $4.50 \%$ & 0.655 \\
\hline CT score 1 in the first time & \multicolumn{2}{|c|}{$2.31 \pm 0.71$} & \multicolumn{2}{|c|}{$2.58 \pm 0.72$} & 0.084 \\
\hline CT score 2 in the first time & \multicolumn{2}{|c|}{$2.30 \pm 0.78$} & \multicolumn{2}{|c|}{$2.63 \pm 0.65$} & 0.053 \\
\hline CT total score in the first time & \multicolumn{2}{|c|}{$4.62 \pm 1.28$} & \multicolumn{2}{|c|}{$5.21 \pm 1.10$} & 0.035 \\
\hline Antiviral therapy & 811 & $99.10 \%$ & 41 & $100.00 \%$ & 0.552 \\
\hline Antibiotic therapy & 484 & $99.40 \%$ & 34 & $100.00 \%$ & 0.646 \\
\hline Anticoagulation treatment & 103 & $6.10 \%$ & 21 & $38.20 \%$ & $<0.001$ \\
\hline Use of corticosteroid & 90 & $5.30 \%$ & 17 & $30.90 \%$ & $<0.001$ \\
\hline Death & 8 & $0.50 \%$ & 7 & $12.70 \%$ & $<0.001$ \\
\hline Follow-up days, mean \pm SD & \multicolumn{2}{|c|}{$19.26 \pm 8.893$} & \multicolumn{2}{|c|}{$22.96 \pm 10.38$} & 0.004 \\
\hline
\end{tabular}

admission or in the highest level of illness severity during hospitalization (both $P<0.001$; Table 1). In addition, more patients in the elevated $\mathrm{LDH}$ group required critical airway management [tracheal intubation or extracorporeal membrane oxygenation (ECMO)] $(P<0.001$; Table 1), and those in the elevated LDH group had significantly higher in-hospital mortality $(12.7 \%)$ than those in the normal/decreased LDH group $(0.50 \%, P<0.001$; Table 1). Patients in the elevated LDH group were also more likely to need anticoagulation treatment and corticosteroids (both $P<0.001$; Table 1).

\section{Laboratory Findings}

As shown in Table 2, there were significant differences in most laboratory indexes according to the $\mathrm{LDH}$ level. The median interleukin-6 in the elevated LDH group was above the normal range and was significantly higher than that in the normal/decreased $\mathrm{LDH}$ group, as was D-dimer, indicating that patients with elevated $\mathrm{LDH}$ had a more intense inflammatory responses and more of these patients were in a hypercoagulable state. In addition, patients with elevated LDH were more likely to have lymphopenia $(P<0.001)$. However, the prevalence of SARS-CoV-2 immunoglobulin $\mathrm{M}$ 
TABLE 2 | Outcomes of laboratory tests for COVID-19 patients in different LDH level.

\begin{tabular}{|c|c|c|c|c|}
\hline Covariate & LDH normal or decreased group $(n=1,696)$ & LDH evaluated group $(n=55)$ & $P$-value & Reference \\
\hline Interleukin-6, pg/mL & $1.50(1.50-3.74)$ & $17.77(4.17-49.24)$ & $<0.001$ & $0.00-7.00$ \\
\hline Procalcitonin, ng/mL & $0.04(0.03-0.05)$ & $0.11(0.06-0.21)$ & $<0.001$ & $<0.05$ \\
\hline Aspartate aminotransferase, $\mathrm{U} / \mathrm{L}$ & $19.00(16.00-26.00)$ & $42.60(31.00-86.00)$ & $<0.001$ & $15.00-40.00$ \\
\hline Albumin, g/L & $37.80(35.10-40.10)$ & 32.80 (30.30-36.20) & $<0.001$ & $40.00-55.00$ \\
\hline Direct bilirubin, $\mu \mathrm{mol} / \mathrm{L}$ & $3.10(2.40-4.20)$ & $4.60(2.60-8.00)$ & $<0.001$ & $0.00-7.00$ \\
\hline Indirect bilirubin, $\mu \mathrm{mol} / \mathrm{L}$ & $5.70(4.30-7.80)$ & $4.80(3.40-7.60)$ & 0.085 & $1.50-1.80$ \\
\hline Creatinine, $\mu \mathrm{mol} / \mathrm{L}$ & $64.10(54.10-76.00)$ & $67.10(57.40-90.00)$ & 0.730 & $64.00-104.00$ \\
\hline Ureanitrogen, mmol/L & $4.80(3.90-5.80)$ & $5.90(4.10-8.19)$ & 0.001 & $2.80-7.60$ \\
\hline INR & $0.97(0.93-1.01)$ & $1.01(0.97-1.10)$ & $<0.001$ & $0.85-1.15$ \\
\hline Fibrinogen, $g / L$ & $2.92(2.51-3.67)$ & $4.08(3.09-4.75)$ & $<0.001$ & $2.38-4.98$ \\
\hline D-dimer, mg/L & $0.37(0.21-0.87)$ & $1.37(0.61-4.07)$ & $<0.001$ & $<0.50$ \\
\hline White blood cell count, $\times 10^{9} / \mathrm{L}$ & $5.68(4.70-6.8)$ & $6.92(5.27-9.40)$ & $<0.001$ & $3.5-9.5$ \\
\hline Neutrophil count, $\times 10^{9} / \mathrm{L}$ & $3.25(2.53-4.23)$ & $4.53(3.35-7.89)$ & $<0.001$ & $1.8-6.3$ \\
\hline Lymphocyte count, $\times 10^{9} / \mathrm{L}$ & $1.62(1.27-1.99)$ & $0.96(0.59-1.35)$ & $<0.001$ & $1.1-3.2$ \\
\hline Monocyte count, $\times 10^{9} / \mathrm{L}$ & $0.50(0.40-0.63)$ & $0.57(0.41-0.74)$ & 0.108 & $0.1-0.6$ \\
\hline Red blood cell count, $\times 10^{9} / \mathrm{L}$ & $4.12(3.77-4.49)$ & $3.90(3.52-4.40)$ & 0.035 & $4.3-5.8$ \\
\hline Hemoglobin, g/L & $126.00(115.00-137.00)$ & $122.00(107.00-134.00)$ & 0.125 & $130-175$ \\
\hline Hematocrit, \% & 38.00 (34.90-40.90) & 36.90 (32.20-40.20) & 0.041 & $40.00-50.00$ \\
\hline Platelet count, $\times 10^{9} / \mathrm{L}$ & 229.00 (188.00-277.00) & 203.00 (141.00-280.00) & 0.020 & $125.00-350.00$ \\
\hline $\operatorname{lgM}(+)$ of SARS-CoV-2 & 199 (35.20\%) & $9(40.90 \%)$ & 0.584 & $(-)$ \\
\hline
\end{tabular}

TABLE 3 | Univariate and multivariate Cox regression analysis for the survival of patients in different LDH level.

\begin{tabular}{|c|c|c|c|c|c|}
\hline \multicolumn{2}{|c|}{ Group } & \multicolumn{4}{|c|}{ Cox regression analysis } \\
\hline & LDH evaluated group & 26.626 & 9.624 & 73.661 & $<0.001$ \\
\hline \multirow[t]{2}{*}{ Multivariate analysis* } & LDH normal or decrease group & ref & & & \\
\hline & LDH evaluated group & 4.491 & 1.218 & 16.560 & 0.024 \\
\hline
\end{tabular}

${ }^{*}$ Adjust for age, the history of cardiovascular disease, WBC, PLT, lymphocyte count, D-Dimer.

TABLE 4 | Univariate and multivariate cox regression analysis for the survival of patients and logistics regression analysis for the severity of patients when taking LDH as a continuous variable.

\begin{tabular}{|c|c|c|c|c|c|c|c|c|c|}
\hline \multicolumn{2}{|c|}{ Group } & \multicolumn{4}{|c|}{ Cox regression analysis } & \multicolumn{4}{|c|}{ Logistics regression analysis } \\
\hline Multivariate analysis* & LDH level & 1.006 & 1.002 & 1.009 & 0.001 & 1.003 & 0.992 & 1.014 & 0.577 \\
\hline
\end{tabular}

*Adjust for age, the history of cardiovascular disease, WBC, PLT, lymphocyte count, D-Dimer. 


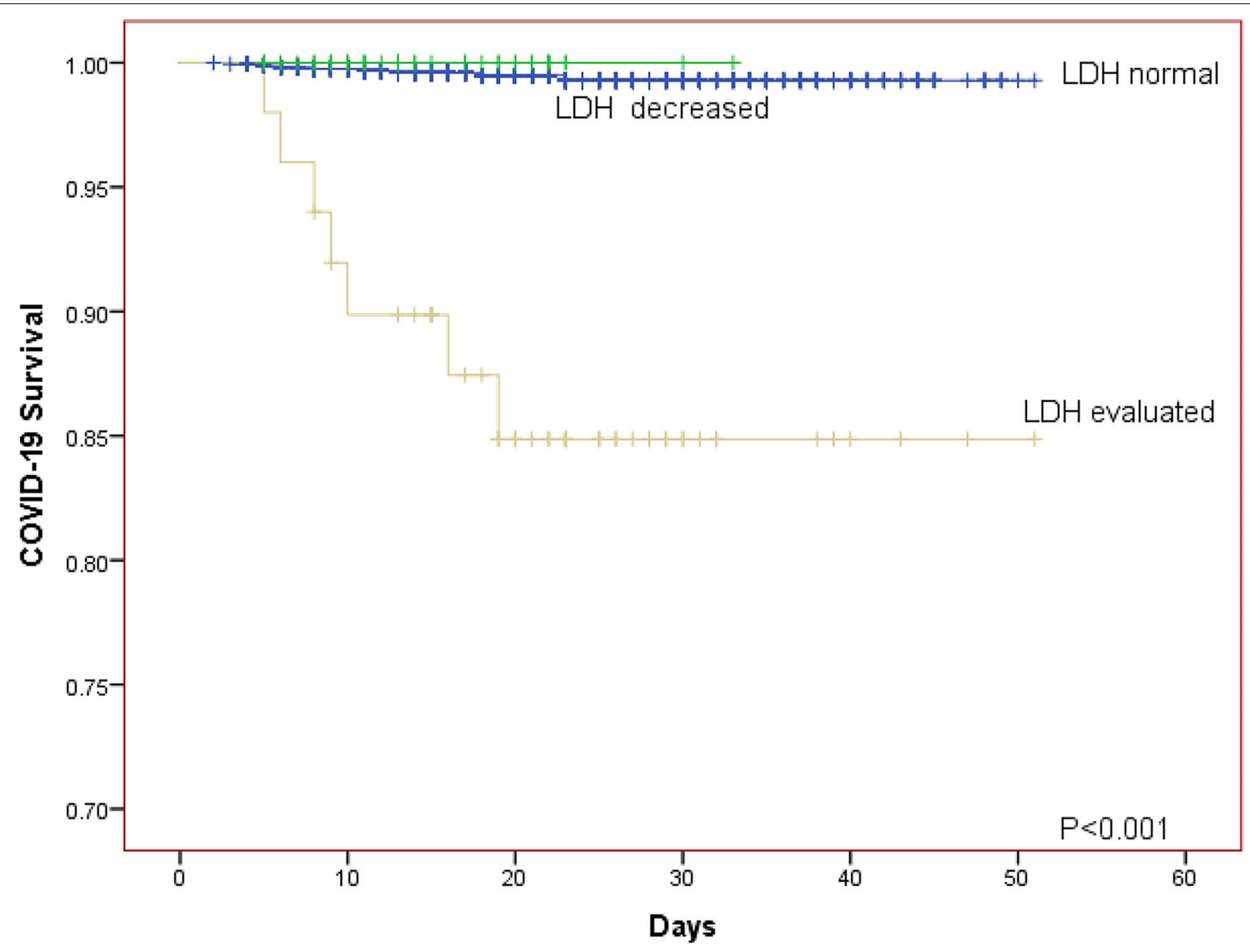

FIGURE 1 | The Kaplan-Meier curves for the survival of COVID-19 patients in different LDH level (normal group, decreased group, evaluated group).

and immunoglobulin $\mathrm{G}$ did not differ significantly according to the $\mathrm{LDH}$ level.

\section{Analysis for the Relationship Between Prognosis and LDH Level}

The univariate Cox regression analysis showed that patients in the elevated $\mathrm{LDH}$ group had a higher risk of in-hospital death than those in the normal or decreased LDH group [hazard ratio (HR): 26.626, 95\% confidence interval (CI): 9.62473.661, $P<0.001$; Table 3]. The result of univariate logistics regression analysis presented the same tendency that elevated LDH group suffered higher risk of developing into sever or critical illness status than those in the normal or decreased $\mathrm{LDH}$ group [odds ratio (OR): 11.216, 95\% CI: 4.447-28.288, $P<$ 0.001; Supplementary Table 1]. The adjustment factors included in the multivariate Cox regression model were age, history of cardiovascular disease, white blood cell count, platelet count, lymphocyte count, and D-dimer. The results of the multivariate analysis showed that an elevated LDH level was an independent risk factor for in-hospital death $(P=0.024$; Table 3). Elevated LDH level was not related to sever or critical illness status after adjustment ( $P=0.997$; Supplementary Table $\mathbf{1})$.

$\mathrm{LDH}$ was taken as a continuous variable in further analysis. The result was similar to the previous analysis which LDH level was divided into groups. LDH was an independent risk factor for in-hospital death (univariate analysis $P<0.001$, multivariate analysis $P=0.001$; Table 4 ) but not for severe or critical illness status (univariate analysis $\mathrm{P}<0.001$, multivariate analysis $P=$ 0.557; Table 4). Each unit increase in LDH level was associated with higher risk of death (univariate analysis: $\mathrm{HR}=1.002$, 95 CI\%: 1.001-1.002; multivariate analysis: $\mathrm{HR}=1.006,95$ CI\%: 1.002-1.009).

The Kaplan-Meier curves was further generated for descripting the relationship between survival of patients and LDH level. The Kaplan-Meier curves for LDH level showed that patients in the elevated $\mathrm{LDH}$ group had worse prognosis than those with normal/decreased LDH regardless of whether the patients were divided into two or three $\mathrm{LDH}$ groups (both $P<0.001$; Figures 1, 2).

\section{Curve Fitting Analysis for the Evaluation of CT Images}

Figure 3 shows the result of the curve fitting analysis for CT images and days that the CT scan was done. Score 1 for all patients reached the peak at 20 days (2.5 points, Figure $3 \mathbf{A}$ ), while score 2 for all patients reached the lowest point at 12 days (2.40 points, Figure 3B). The total score reached the peak at 19 days (4.90 points, Figure 3C). Similarly, score 1 for patients 


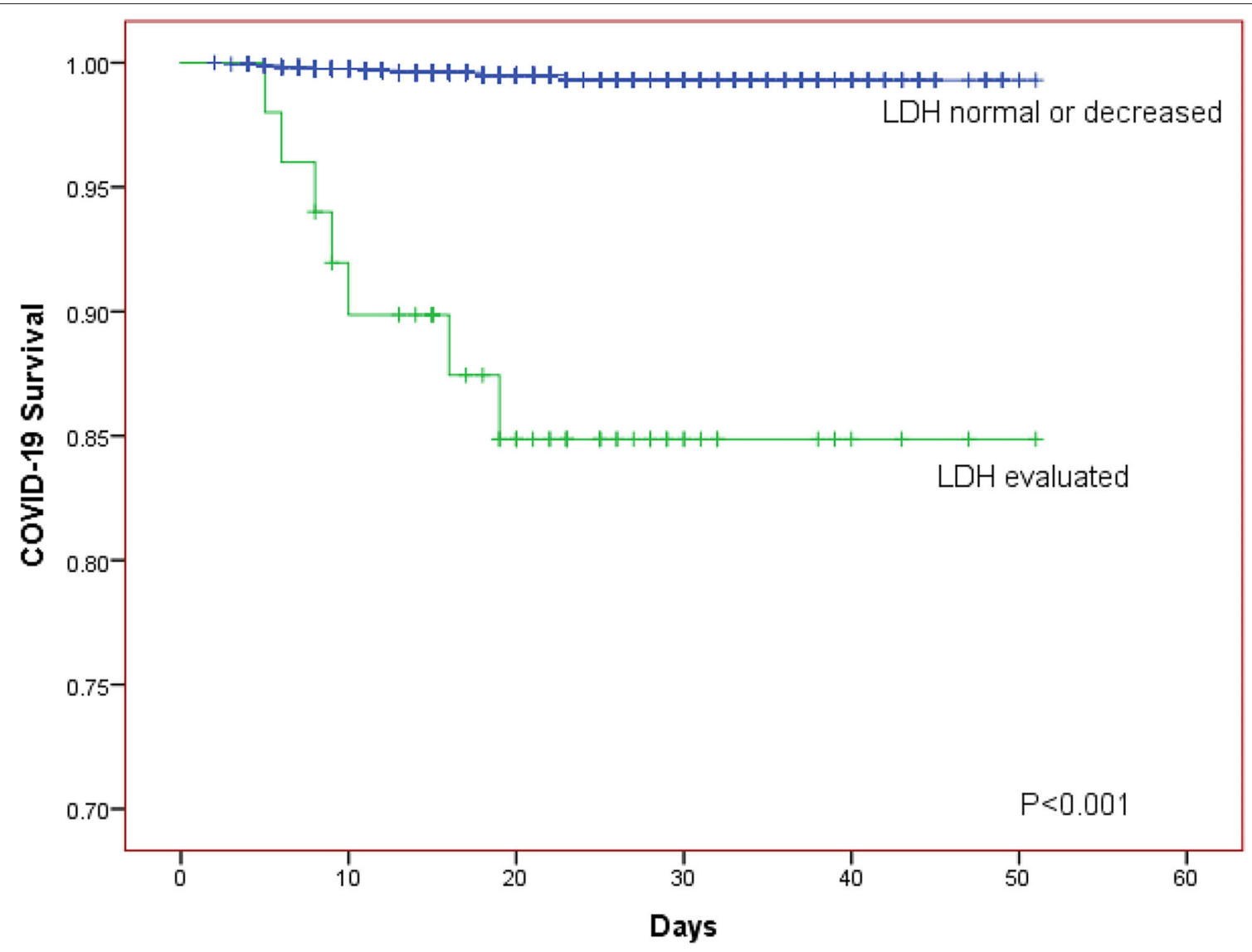

FIGURE 2 | The Kaplan-Meier curves for the survival of COVID-19 patients in different LDH level (normal or decreased group, evaluated group).

with normal/decreased $\mathrm{LDH}$ reached the peak of 2.42 at 21 days (Figure 3D), score 2 reached the peak of 2.30 at 16 days (Figure 3E) and total score reached the peak of 4.70 at 20 days (Figure 3F). For patients with evaluated level of LDH, score 1, and total score reached the peak of 2.90, 5.80 on 19, 16 days, respectively (Figures 3G,I). However, the tendency of score 2 for patients with elevated LDH level tended to be descending (Figure 3H).

\section{DISCUSSION}

We investigated the effect of $\mathrm{LDH}$ on the clinical course and survival of patients with laboratory-confirmed COVID-19 based on a large sample with 1,880 patients and found that patients with elevated LDH were associated with higher mortality on univariate or multivariate Cox regression analysis no matter $\mathrm{LDH}$ was taken as classified variable or continuous variable. The Kaplan-Meier curves for COVID-19 progress also showed the same tendency.

In addition to the reticulocyte count, indirect bilirubin levels, serum haptoglobin, and LDH levels have been used as markers of hemolysis (5). In another study, Tasaka et al. (12) found that measuring $\mathrm{LDH}$ levels could help improve the diagnosis of pneumocystis pneumonia. Furthermore, they found that the HIV-positive patients had higher LDH levels than HIV-negative patients. These studies reveal that $\mathrm{LDH}$ plays an important role in differentiating disease, including that of the immune system. In our study, although we did not compare the LDH levels in patients with COVID-19 with the LDH levels in patents with other types of pneumonia or the normal population, an elevated LDH level was predictive of higher mortality in patients with COVID-19. Therefore, LDH was shown to be associated with disease diagnosis and prognosis.

COVID-19 patients with higher LDH levels tended to be older, and were more likely to require respiratory support. On the other hand, the patients in the elevated LDH group had similar comorbidities to the other patients. In patients with pneumonia, the presence of comorbidities may adversely affect the clinical course and the outcome (13). In our cohort, the prevalence of pulmonary disease did not differ according to the LDH level; therefore, the comorbidities did not act as confounders of the association between LDH levels and survival in patients with COVID-19. Previous study found that the levels of LDH in severe cases of COVID-19 were significantly higher than both nonsevere cases of COVID-19 and healthy control group, while the $\mathrm{LDH}$ level of non-severe cases were also higher than healthy group (14). In this study, LDH level was associated with severe or critical illness status in univariate logistics regression analysis, which was in accordance with the result of previous study. 
D
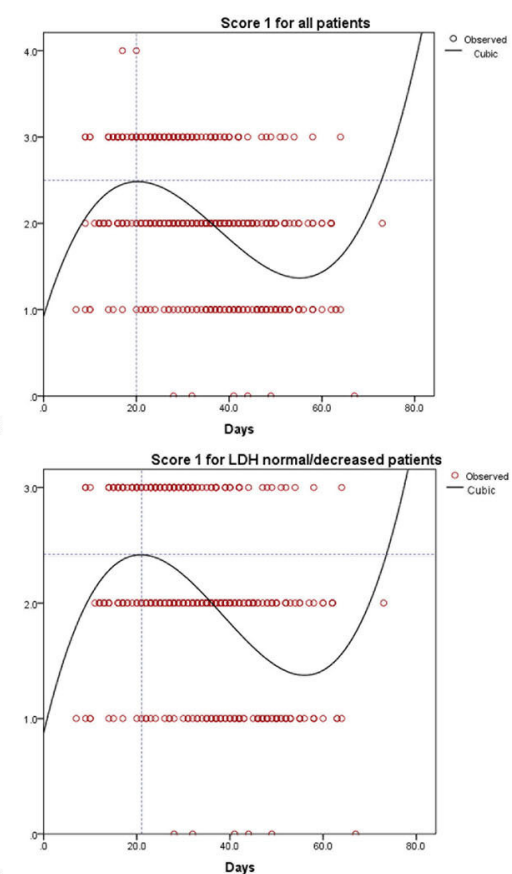

G

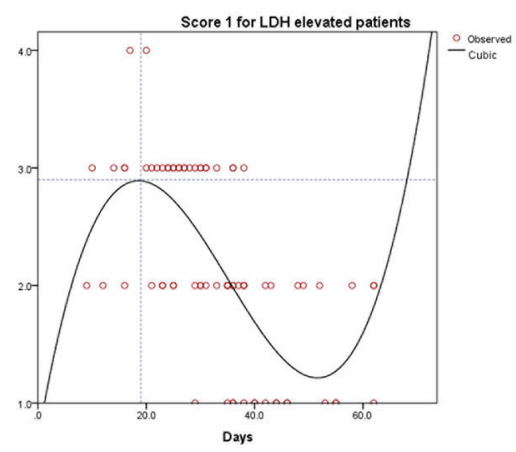

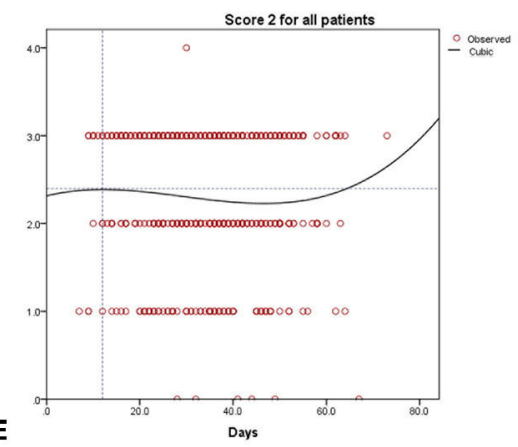

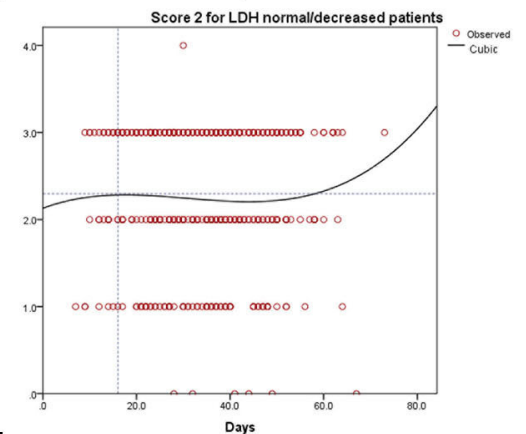

H

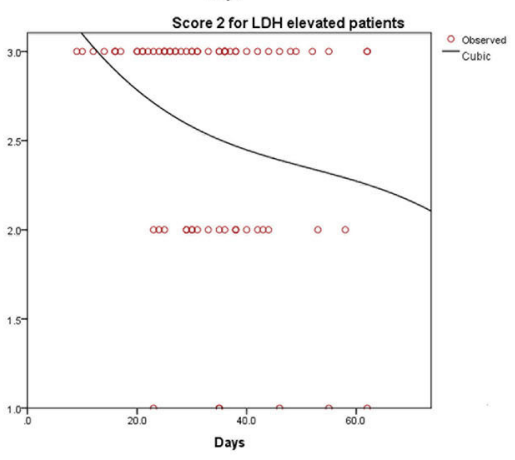

C
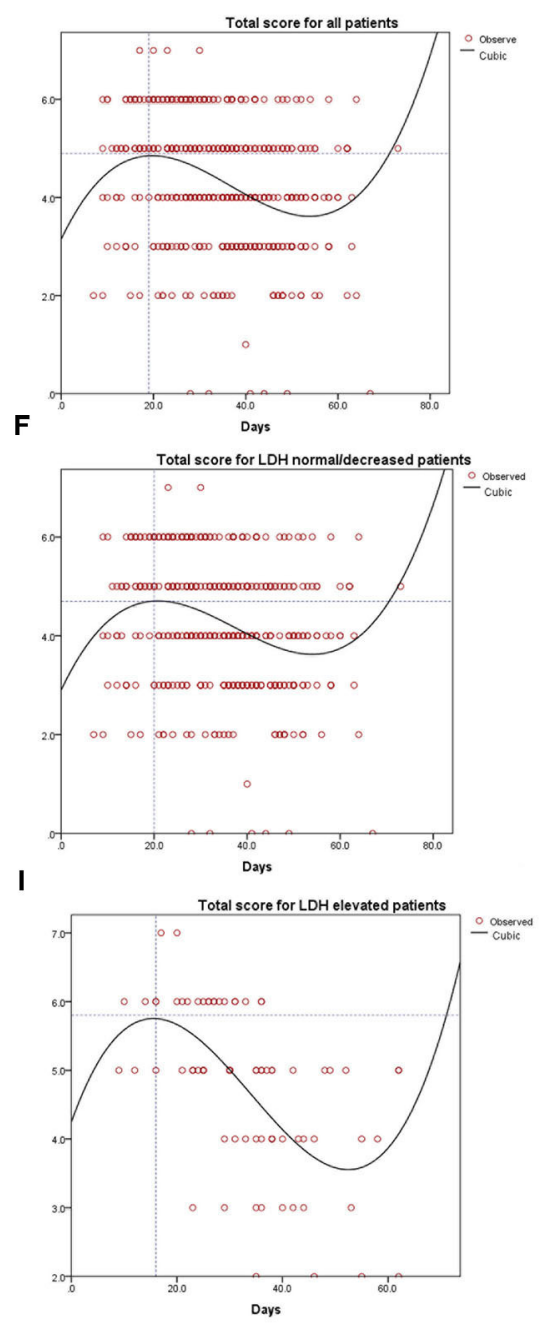

FIGURE 3 | Curve fitting analysis for all the COVID-19 patients (A-C), LDH normal or decreased group (D-F) and LDH evaluated group (G-I).

However, significant differences were not found in multivariate regression analysis which contained adjustment of confounding factors including age, the history of cardiovascular disease, WBC, PLT, lymphocyte count, D-Dimer. We hypothesized that elder patients and patients with the history of cardiovascular disease essentially burden higher risk of cardiac muscle or lung interstitial damage.

In the early phase of COVID-19, CT images reveal multifocal peripheral and basal ground-glass opacities, crazy paving patterns, traction bronchiectasis, and air bronchogram signs $(15,16)$. However, depending on various factors, such as the evolution of the disease course or severity, comorbidity and therapy, CT presentations are dynamic and manifestation patterns often overlap (13, 17-19). With the progression of the clinical course, the CT manifestations include pleural effusion, irregular interlobular, and septal thickening (16). In this study, the CT manifestations were evaluated and presented as score
1 (imaging feature type), score 2 (lesion distribution), and the total score (score 1 plus score 2) by two independent radiologists to record the dynamic changes (20). Fitting curve for imaging manifestations types of lung inflammation and lesion distribution in normal or lower $\mathrm{LDH}$ group showed a trend of first rise then descend, however, higher LDH group patients showed a trend of rapidly rising and then rapidly falling (Figures 3G,I) or presented as a trend of declining all along (Figure 3H). This may be because patients with elevated LDH tended to have severe clinical symptoms of pneumonia and were then transported to the hospital for unified and timely medical treatment.

In addition, the use of antiviral therapy and antibiotic therapy did not differ according to the LDH level among the patients in our study. However, a higher proportion of patients with elevated $\mathrm{LDH}$ received anticoagulation treatment and corticosteroid. Drug treatment, especially the use of corticosteroids, may 
slow virus clearance due to its immunosuppressive effect $(21,22)$. This may affect the disease course and biochemical indicators, including LDH; therefore, further research is needed to determine the effects of corticosteroids and anticoagulants on LDH in patients with COVID-19.

Other studies have found that an elevated $\mathrm{LDH}$ level is a sensitive biomarker for lymphoproliferative disorders $(23,24)$. Ghobrial et al. (25) and Boothpur et al. (26) identified that serum $\mathrm{LDH}$ was one of the negative prognostic factors for overall survival and recurrence. Many studies have found a significant drop in T lymphocyte subsets and an increase in inflammatory cytokines in patients with COVID-19 $(8,27)$. Nguyen et al. (24) demonstrated that the SARS-CoV-2 virus could enable crossprotective T-cell based immunity in a comprehensive in silico analysis. In our study, patients in the elevated $\mathrm{LDH}$ group had a higher white blood cell count but lower lymphocyte count than patients with normal/decreased LDH. Overwhelming inflammation and cytokine-associated lung injury could be important factors in initiating severe events in patients with COVID-19 (28), Therefore, LDH may affect the clinical course of COVID-19 by causing inflammation and lung injury, and influencing $\mathrm{T}$-cell based immunity.

Jiang et al. found that patients with COVID-19 had IgG and IgM antibodies which specifically combine with SARS$\mathrm{CoV}-2$ proteins, particularly the $\mathrm{N}$ protein and $\mathrm{S} 1$ protein (29). They also found that S1 specific IgG signal positively correlates with the level of LDH (29). However, whether serum lactate dehydrogenase have any similar physiological function or pathological pathway to affect the clearance of SARS-CoV-2 remains unclear and warrants further study.

This study has some limitations. Heterogeneity is an unavoidable limitation of retrospective studies Data of patients were collected retrospectively, which inevitably led to biases in our study. Another limitation is a lack of research on the mechanism of serum lactate dehydrogenase levels as a common risk factor for COVID-19 progress and prognosis. In addition, the role of drug interference such as glucocorticoids, antiviral and antibacterial treatment cannot be excluded. Further multicenter prospective studies with a larger sample size are needed to verify the findings and to determine the pathogenic mechanism by which LDH exerts an effect on patients with COVID-19.

\section{REFERENCES}

1. Zhu N, Zhang D, Wang W, Li X, Yang B, Song J, et al. A novel coronavirus from patients with pneumonia in China, 2019. N Engl J Med. (2020) 382:72733. doi: 10.1056/NEJMoa2001017

2. Zhao D, Yao F, Wang L, Zheng L, Gao Y, Ye J, et al. A comparative study on the clinical features of COVID-19 pneumonia to other pneumonias. Clin Infect Dis. (2020) 71:756-61. doi: 10.1093/cid/ciaa247

3. Liang W, Guan W, Chen R, Wang W, Li J, Xu K, et al. Cancer patients in SARS-CoV-2 infection: a nationwide analysis in China. Lancet Oncol. (2020) 21:335-7. doi: 10.1016/S1470-2045(20)30096-6

4. Guan WJ, Ni ZY, Hu Y, Liang WH, Ou CQ, He JX, et al. Clinical characteristics of coronavirus disease 2019 in China. N Engl J Med. (2020) 382:170820. doi: 10.1056/NEJMoa2002032

\section{CONCLUSION}

Our study revealed that $\mathrm{LDH}$ level is an independent risk factor for the survival of patients with COVID-19 and a high LDH level is a predictor of mortality in patients with COVID-19. However, LDH level seems not to be associated with severe or critical illness status. This study will provide a valuable reference for clinicians and researchers to understand, diagnose, and treat patients with COVID-19, although prospective studies with a larger sample size are needed to verify the findings and to determine the pathogenic mechanism by which LDH exerts an effect on patients with COVID-19.

\section{DATA AVAILABILITY STATEMENT}

The original contributions presented in the study are included in the article/Supplementary Material, further inquiries can be directed to the corresponding author/s.

\section{ETHICS STATEMENT}

This study obtained the approval of the Research Ethics Commission of the Zhongnan Hospital of Wuhan University (approval number: 2020074). The requirement for informed consent was waived because the study was retrospective.

\section{AUTHOR CONTRIBUTIONS}

YH, ZLiu, and JL: conception and design. LG, KLu, and XW: administrative support. KLi and ZX: provision of study materials or patients. QW and JC: collection and assembly of data. $\mathrm{YH}, \mathrm{CZ}$, and ZLi: data analysis and interpretation. $\mathrm{YH}$ and ZLiu: manuscript writing. MW, WY, and XW: final approval of manuscript. All authors contributed to the article and approved the submitted version.

\section{SUPPLEMENTARY MATERIAL}

The Supplementary Material for this article can be found online at: https://www.frontiersin.org/articles/10.3389/fmed. 2021.671667/full\#supplementary-material

5. Ballas SK. Lactate dehydrogenase and hemolysis in sickle cell disease. Blood. (2013) 121:243-4. doi: 10.1182/blood-2012-10-462135

6. Guo G, Sun L, Yang L, Xu H. IDO1 depletion induces an antiinflammatory response in macrophages in mice with chronic viral myocarditis. Cell Cycle. (2019) 18:2598-613. doi: 10.1080/15384101.2019.16 752471

7. Dennison JB, Molina JR, Mitra S, Gonzalez-Angulo AM, Balko JM, Kuba MG, et al. Lactate dehydrogenase B: a metabolic marker of response to neoadjuvant chemotherapy in breast cancer. Clin Cancer Res. (2013) 19:370313. doi: 10.1158/1078-0432.CCR-13-0623

8. Liu Z, Long W, Tu M, Chen S, Huang Y, Wang S, et al. Lymphocyte subset $(\mathrm{CD} 4+, \mathrm{CD} 8+)$ counts reflect the severity of infection and predict the clinical outcomes in patients with COVID-19. J Infect. (2020) 81:31856. doi: 10.1016/j.jinf.2020.03.054 
9. Vincent JL, Taccone FS. Understanding pathways to death in patients with COVID-19. Lancet Respir Med. (2020) 8:4302. doi: 10.1016/S2213-2600(20)30165-X

10. Huang $\mathrm{Y}, \mathrm{Tu} \mathrm{M}$, Wang $\mathrm{S}$, Chen S, Zhou W, Chen D, et al. Clinical characteristics of laboratory confirmed positive cases of SARS-CoV-2 infection in Wuhan, China: a retrospective single center analysis. Travel Med Infect Dis. (2020) 36:101606. doi: 10.1016/j.tmaid.2020.101606

11. China NHCotPsRo. The Notice of Launching Guideline on Diagnosis and Treatment of the Novel Coronavirus Pneumonia (NCP). Revised version of the 7th edition (2020). Available online at: https://www.chinacdc.cn/jkzt/crb/zl/ szkb_11803/jszl_11815/202003/t20200305_214142.html

12. Tasaka S, Hasegawa N, Kobayashi S, Yamada W, Nishimura T, Takeuchi T, et al. Serum indicators for the diagnosis of pneumocystis pneumonia. Chest. (2007) 131:1173-80. doi: 10.1378/chest.06-1467

13. Duan YN, Qin J. Pre- and posttreatment chest CT findings: 2019 novel coronavirus (2019-nCoV) pneumonia. Radiology. (2020) 295:21. doi: 10.1148/radiol.2020200323

14. Jin Z, Zheng M, Shi J, Ye X, Cheng F, Chen QL, et al. Correlation analysis between serum uric acid, prealbumin level, lactate dehydrogenase, and severity of COVID-19. Front Mol Biosci. (2021) 8:615837. doi: $10.3389 /$ fmolb.2021.615837

15. Bernheim A, Mei X, Huang M, Yang Y, Fayad ZA, Zhang N, et al. Chest CT findings in coronavirus disease-19 (COVID-19): relationship to duration of infection. Radiology. (2020) 2020:200463. doi: 10.1148/radiol.2020200463

16. Pan F, Ye T, Sun P, Gui S, Liang B, Li L, et al. Time course of lung changes on chest CT during recovery from 2019 novel coronavirus (COVID-19) pneumonia. Radiology. (2020) 295:715-21. doi: 10.1148/radiol.2020200370

17. Shi H, Han X, Zheng C. Evolution of CT manifestations in a patient recovered from 2019 novel coronavirus (2019-nCoV) pneumonia in Wuhan, China. Radiology. (2020) 295:20. doi: 10.1148/radiol.2020200269

18. Zhang R, Ouyang H, Fu L, Wang S, Han J, Huang K, et al. CT features of SARS-CoV-2 pneumonia according to clinical presentation: a retrospective analysis of 120 consecutive patients from Wuhan city. Eur Radiol. (2020) 30:4417-26. doi: 10.1007/s00330-020-06854-1

19. Song F, Shi N, Shan F, Zhang Z, Shen J, Lu H, et al. Emerging 2019 novel coronavirus (2019-nCoV) pneumonia. Radiology. (2020) 295:2107. doi: 10.1148/radiol.2020200274

20. Zhao W, Zhong Z, Xie X, Yu Q, Liu J. CT scans of patients with 2019 novel coronavirus (COVID-19) pneumonia. Theranostics. (2020) 10:460613. doi: 10.7150/thno. 45016

21. Fang X, Mei Q, Yang T, Li L, Wang Y, Tong F, et al. Low-dose corticosteroid therapy does not delay viral clearance in patients with COVID-19. J Infect. (2020) 81:147-78. doi: 10.1016/j.jinf.2020.03.039

22. Li X, Xu S, Yu M, Wang K, Tao Y, Zhou Y, et al. Risk factors for severity and mortality in adult COVID-19 inpatients in Wuhan. J Allergy Clin Immunol. (2020) 146:110-8. doi: 10.1016/j.jaci.2020.04.006
23. Elstrom RL, Andreadis C, Aqui NA, Ahya VN, Bloom RD, Brozena SC, et al. Treatment of PTLD with rituximab or chemotherapy. Am J Transplant. (2006) 6:569-76. doi: 10.1111/j.1600-6143.2005.0 1211.x

24. Nguyen A, David JK, Maden SK, Wood MA, Weeder BR, Nellore A et al. Human Leukocyte Antigen Susceptibility Map for Severe Acute Respiratory Syndrome Coronavirus 2. J Virol. (2020) 94:e00510-20. doi: 10.1128/JVI.00510-20

25. Ghobrial IM, Habermann TM, Maurer MJ, Geyer SM, Ristow KM, Larson TS, et al. Prognostic analysis for survival in adult solid organ transplant recipients with post-transplantation lymphoproliferative disorders. J Clin Oncol. (2005) 23:7574-82. doi: 10.1200/JCO.2005.0 1.0934

26. Boothpur R, Brennan DC. Didactic lessons from the serum lactate dehydrogenase posttransplant: a clinical vignette. Am J Transplant. (2008) 8:862-5. doi: 10.1111/j.1600-6143.2008.02151.x

27. Xu B, Fan CY, Wang AL, Zou YL, Yu YH, He C, et al. Suppressed T cell-mediated immunity in patients with COVID-19: a clinical retrospective study in Wuhan, China. J Infect. (2020) 81:e51-60. doi: 10.1016/j.jinf.2020. 04.012

28. Xu Z, Shi L, Wang Y, Zhang J, Huang L, Zhang C, et al. Pathological findings of COVID-19 associated with acute respiratory distress syndrome. Lancet Respir Med. (2020) 8:420-2. doi: 10.1016/S2213-2600(20)30076-X

29. Jiang HW, Li Y, Zhang HN, Wang W, Yang X, Qi H, et al. SARS-CoV2 proteome microarray for global profiling of COVID-19 specific IgG and IgM responses. Nat Commun. (2020) 11:3581. doi: 10.1038/s41467-020-1 7488-8

Conflict of Interest: The authors declare that the research was conducted in the absence of any commercial or financial relationships that could be construed as a potential conflict of interest.

Publisher's Note: All claims expressed in this article are solely those of the authors and do not necessarily represent those of their affiliated organizations, or those of the publisher, the editors and the reviewers. Any product that may be evaluated in this article, or claim that may be made by its manufacturer, is not guaranteed or endorsed by the publisher.

Copyright (๑ 2022 Huang, Guo, Chen, Wu, Zhang, Liu, Li, Li, Xiong, Wu, Li, Luo, Yuan and Wu. This is an open-access article distributed under the terms of the Creative Commons Attribution License (CC BY). The use, distribution or reproduction in other forums is permitted, provided the original author(s) and the copyright owner(s) are credited and that the original publication in this journal is cited, in accordance with accepted academic practice. No use, distribution or reproduction is permitted which does not comply with these terms. 